\title{
Sinopsis de la Situación de Salud Oral en Chile - Parte II: Diagnósticos Poblacionales de Salud Oral.
}

\section{Synopsis or the Oral Health Situation in Chile - Part II: Oral Health Surveys.}

\author{
Alicia Morales ${ }^{1,2^{\star}}$, Gisela Jara ${ }^{1,2}$, Fabiola Werlinger ${ }^{1,2}$, Rodrigo Cabello ${ }^{1,2}$, \\ Iris Espinoza ${ }^{1,2}$, Rodrigo Giacamann, ${ }^{2,3}$, Ximena Lee ${ }^{1,2}$, Paola Carvajal ${ }^{1,2}$, Oscar Arteaga ${ }^{2,4}$, \\ Franco Cavalla ${ }^{1,2}$, Camila Corral ${ }^{1,2}$, Mauricio Baeza ${ }^{1,2}$, Gonzalo Rodríguez ${ }^{1,2}$, \\ Soraya León ${ }^{2,3}$, Karla Gambetta ${ }^{2,3}$, Jorge Gamonal ${ }^{1,2^{*}}$
}

\begin{abstract}
1. Facultad de Odontología, Universidad de Chile, Chile.

2. Centro de Vigilancia y Epidemiología de las

Enfermedades Orales - CEVEO, Chile.

3. Universidad de Talca, Chile

4. Escuela de Salud Pública, Universidad de Chile, Chile.
\end{abstract}

* Correspondencia Autor: Alicia Morales | Dirección: Sergio Livingstone 943, comuna de Independencia, Santiago, Chile. | Teléfono: (56) 2 29776772. | E-mail: amorales@odontologia.uchile.cl Jorge Gamonal | Dirección: Sergio Livingstone 943, comuna de Independencia, Santiago, Chile. | Teléfono: (56) 2 29776772. | E-mail: jgamonal@ odontologia.uchile.cl

Trabajo recibido el 27/03/2020.

Aprobado para su publicación el 03/06/2020

\section{RESUMEN}

Objetivo: Sintetizar los resultados sobre los estudios epidemiológicos de caries dental, enfermedad periodontal, desdentamiento y lesiones de mucosa oral de base poblacional con representatividad nacional y regional en adultos chilenos ( $\geq 15$ años). Materiales $y$ métodos: Se realizó una revisión narrativa para identificar aquellos estudios de diagnóstico de salud bucal, a nivel nacional y/o regional, en población adulta de Chile ( $\geq 15$ años), con el objetivo de establecer prevalencias para las patologías bucales de mayor relevancia nacional. Resultados: Se identificaron 6 estudios de representatividad nacional y 7 estudios de representatividad regional. Se reporta una disminución en la prevalencia de caries cavitadas y de dentición no funcional, correspondiendo a un $54.6 \%$ y $27.0 \%$, respectivamente. La prevalencia de pérdida de inserción clínica $\geq 4 \mathrm{~mm}$., es cercana al $100 \%$. La lesión de mucosa oral más prevalente fue la estomatitis subprotésica $(22.3 \%)$. Se observaron inequidades socieconómicas y culturales en la distribución de las patologías orales en la población adulta chilena. Conclusiones: Existe una alta prevalencia de enfermedad periodontal, caries, desdentamiento y lesiones de mucosa oral en adultos y adultos mayores chilenos.

PALABRAS CLAVE:

Salud bucal; Diagnóstico de salud; Prevalencia.

Int. J. Inter. Dent Vol. 13(2); 88-94, 2020.

\section{ABSTRACT}

Aim: To synthesize results of epidemiologic national and regional studies about dental caries, periodontal diseases, tooth loss and oral mucosa lesions in Chilean adults $(\geq 15$ years- old). Methods: A narrative revision was made in order to identify epidemiologic national or regional studies in Chilean adults ( $\geq 15$ years- old). The objective was to establish the prevalence of the most common oral diseases. Results: Six national and seven regional studies were identified. The prevalence of non-treated caries and non- functional dentition was reduced to $54.6 \%$ and $27.0 \%$, respectively. The prevalence of periodontal attachment loss $\geq 4 \mathrm{~mm}$. was almost $100 \%$. The most frequent oral mucosa lesion was denture stomatitis $(22.3 \%)$. Socioeconomic and cultural disparities were observed in the distribution of oral diseases in Chilean adults. Conclusions: The prevalence of dental caries, periodontal diseases, tooth loss and oral mucosa lesions was high in Chilean adults and elderly people.. KEY WORDS:

Oral health; Health survey; Prevalence.

Int. J. Inter. Dent Vol. 13(2); 88-94, 2020.

\section{INTRODUCCIÓN}

Las enfermedades bucales son las más comunes de las enfermedades crónicas y son un importante problema de Salud Pública por su alta prevalencia ${ }^{(1,2)}$, impacto en la calidad de vida de los individuos ${ }^{(3)}$ y el costo de su tratamiento(4). En el año 2017, 3.500 millones de personas a nivel mundial padecían una condición bucal no tratada, lo que corresponde a un $45 \%$ de la población total. La prevalencia de caries no tratada ascendía a un $29.4 \%$ (2.500 millones de personas), de periodontitis severa a un $9.8 \%$ (796 millones de personas) y de pérdida total de dientes a un $3.3 \%$ (267 millones de personas) $)^{(2)}$. Por otro lado, el cáncer de cavidad oral y labios presentó 500.550 casos incidentes a nivel mundial en el año $2018^{(5)}$. El costo directo de tratamiento odontológico asciende a USD 2.976 millones, representando el $4.6 \%$ del gasto total en salud, y los costos indirectos asociados a la pérdida de productividad son de USD 1.442 millones, donde USD 6.303 millones se asocian a pérdida severa de dientes, USD 5.399 millones a periodontitis severa y USD 2.514 millones a caries no tratada $^{(6)}$. Es relevante destacar que las enfermedades orales comparten factores de riesgo y han sido asociadas a múltiples enfermedades crónicas no transmisibles, por lo que el estudio de su distribución, así como la implementación de intervenciones para controlarlas podría tener 
efectos benéficos en el control de las complicaciones asociadas a otras patologías ${ }^{(7,8)}$.

En Chile, han sido cruciales los estudios epidemiológicos del estado de salud general y oral para apoyar el proceso de reforma en salud que se inició el año 2000. Para ello, se ha requerido disponer de un diagnóstico actualizado para evaluar y reformular las políticas sectoriales, estimar la demandas que enfrentará el sistema de salud y establecer la vigilancia epidemiológica correspondiente. El objetivo de este estudio ha sido sintetizar los resultados de los estudios epidemiológicos de caries dental, enfermedad periodontal, desdentamiento y lesiones de mucosa oral de base poblacional con representatividad nacional y regional en adultos chilenos ( $\geq 15$ años), así como los indicadores de riesgo para cada una de estas patologías, de modo de sintetizar la información y permitir la difusión y comunicación de estos resultados entre clínicos y académicos.

\section{MATERIALES Y MÉTODOS}

Se realizó una revisión narrativa para identificar aquellos estudios de diagnóstico de salud bucal, a nivel nacional y/o regional. Los criterios de inclusión consideraron estudios de base poblacional, con datos primarios y secundarios, con el objetivo de establecer prevalencias para las patologías bucales de mayor relevancia nacional (caries dental, enfermedad periodontal, desdentamiento, lesiones de la mucosa oral) en población adulta chilena ( $\geq 15$ años). La búsqueda incluyó repositorios electrónicos científicos (Pubmed y Scielo) y fuentes de referencia nacional en salud (Ministerio de Salud) y universidades.

\section{RESULTADOS}

Se identificó un total de seis estudios de representatividad nacional (Tabla 1). Dos de ellos incluyeron las temáticas de salud oral dentro de la medición de otros problemas de salud ${ }^{(9,10)}$, dos analizaron datos secundarios $^{(11,12)}$, y dos de ellos corresponden al mismo proyecto de investigación ${ }^{(13,14)}$ analizado con criterios distintos.

Se identificaron siete estudios a nivel regional (Tabla 2), en la Región
Metropolitana $(n=2)^{(15,16)}$, Valparaíso $(n=1)(17)$ y del Maule $(n=4)^{(18-21)}$.

Estudios poblacionales de diagnóstico de caries (Tabla 3)

Se identificaron tres estudios a nivel nacional $\left.\right|^{(9,10,14)}$. La prevalencia de caries cavitadas en las personas $\geq 17$ años fue de un $70 \%$ en el año $2003^{(9,22)}$, disminuyendo significativamente a un $54.6 \%$ en los años 2016 2017 , donde la prevalencia de caries cavitadas fue mayor en los hombres que en las mujeres, y en grupos de mayor edad ${ }^{(10)}$. Al analizar el COPD en los adultos de 35 a 44 años y de 65 a 74 años, éste fue de 15.06 y 21.57 , respectivamente. La media de dientes con caries fue mayor en hombres en el grupo de 35 a 44 años y en el de 65 a 74 años, pero en el de 35- 44 años, la media de dientes obturados sin caries fue mayor en el género femenino(14).

Se identificaron 3 estudios a nivel regional(17-19). En la Región de Valparaíso, el COPD en los adultos $\geq 60$ años fue de un 21.6. Los indicadores de riesgo asociados a la historia de caries de esta población fueron la edad, bajo nivel de ingreso, ser soltero/ viudo, indigente, y de localidad rural(17). En la Región del Maule, en los adultos de 65 a 74 años, el COPD fue de 25.7, donde las necesidades de tratamiento restaurador fue mayor en los hombres y en aquellos con $\leq 8$ años de educación ${ }^{(18)}$. En este grupo de edad, el $64.1 \%$ de las superficies radiculares se encontraban con recesión gingival, el índice de caries radicular fue de un $8.23 \%$, con 0.21 y 0.55 de las superficies obturadas y con caries, respectivamente. Esto indica que la historia de caries tuvo un puntaje de 0.75 , y la mayoría de las superficies presentaban caries $(72.3 \%)$. Las personas mayores con educación secundaria y que vivían en comunidades con agua potable, presentaron un mayor promedio de superficies radiculares obturadas ${ }^{(19)}$.

Estudios poblacionales de diagnóstico de Enfermedades Periodontales (Tabla 4)

A nivel nacional, se identificó un estudio en sujetos de 35 a 44 años y 65 a 74 años. La prevalencia de pérdida de inserción clínica $\geq 4 \mathrm{~mm}$., en el grupo de menor edad fue de un $93.45 \%$ y de un $97.58 \%$ en el grupo etario mayor. Cuando el umbral es de pérdida de inserción clínica $\geq 7 \mathrm{~mm}$., la prevalencia en los sujetos de 35 a 44 años fue de un $38.65 \%$ y de un $69.35 \%$ en los de 65 a 74 años. Los indicadores de riesgo para pérdida de

Tabla 1. Estudios poblacionales de diagnóstico de salud oral de alcance Nacional.

\begin{tabular}{|c|c|c|c|c|c|c|c|c|}
\hline Autor & Alcance & Tipo de estudio & $\begin{array}{c}\text { Población } \\
\text { objetivo }\end{array}$ & Financiamiento & $\begin{array}{c}\text { Año de } \\
\text { desarrollo }\end{array}$ & $\begin{array}{c}\text { Tipo de } \\
\text { publicación }\end{array}$ & Objetivo & $\begin{array}{c}\text { Evento de salud } \\
\text { oral }\end{array}$ \\
\hline $\begin{array}{l}\text { MINSAL, } \\
\text { PUC. } 2003\end{array}$ & Nacional & $\begin{array}{c}\text { Encuesta } \\
\text { poblacional de } \\
\text { tipo transversal. } \\
\text { Examen dental } \\
\text { realizado por } \\
\text { enfermera } \\
\end{array}$ & $\begin{array}{l}\text { Personas } \geq 17 \\
\text { años }(n=3619)\end{array}$ & MINSAL & 2003 & Informe técnico & $\begin{array}{l}\text { Determinar la prevalencia } \\
\text { de las enfermedades } \\
\text { prioritarias y su } \\
\text { variabilidad, en población } \\
\text { adulta chilena }\end{array}$ & $\begin{array}{c}\text { Caries dental y } \\
\text { desdentamiento }\end{array}$ \\
\hline $\begin{array}{l}\text { Riera y } \\
\text { Martínez, } \\
2005\end{array}$ & Nacional & $\begin{array}{l}\text { Estudio } \\
\text { ecológico }\end{array}$ & $\begin{array}{c}\text { Defunciones } \\
\text { obtenidas del } \\
\text { Instituto Nacional } \\
\text { de Estadística } \\
\text { de Chile y } \\
\text { Organización } \\
\text { Mundial de la } \\
\text { Salud } \\
\end{array}$ & MINSAL & 1955- 2002 & $\begin{array}{l}\text { Artículo de } \\
\text { Investigación }\end{array}$ & $\begin{array}{l}\text { Describir la mortalidad y } \\
\text { morbilidad por cáncer oral } \\
\text { y faríngeo en Chile entre } \\
\text { los años 1955- } 2002\end{array}$ & $\begin{array}{l}\text { Mortalidad y } \\
\text { morbilidad por cáncer } \\
\text { oral y faríngeo }\end{array}$ \\
\hline $\begin{array}{l}\text { Gamonal et } \\
\text { al, } 2010\end{array}$ & Nacional & $\begin{array}{l}\text { Estudio de } \\
\text { prevalencia }\end{array}$ & $\begin{array}{l}\text { Adultos de } 35-44 \\
(n=1092) \text { y } 65-74 \\
\text { años }(n=469)\end{array}$ & Fondecyt, Conicyt & 2007- 2009 & $\begin{array}{l}\text { Artículo de } \\
\text { Investigación }\end{array}$ & $\begin{array}{c}\text { Determinar la prevalencia, } \\
\text { extensión y severidad } \\
\text { de la enfermedad } \\
\text { periodontal, y sus } \\
\text { indicadores de riesgo } \\
\text { asociados }\end{array}$ & $\begin{array}{c}\text { Enfermedad } \\
\text { periodontal y } \\
\text { desdentamiento }\end{array}$ \\
\hline $\begin{array}{l}\text { Urzúa et al, } \\
2012\end{array}$ & Nacional & $\begin{array}{l}\text { Estudio de } \\
\text { prevalencia }\end{array}$ & $\begin{array}{l}\text { Adultos de } 35-44 \\
(n=1088) \text { y } 65-74 \\
\text { años }(n=465)\end{array}$ & Fondecyt, Conicyt & 2007- 2009 & $\begin{array}{l}\text { Artículo de } \\
\text { Investigación }\end{array}$ & $\begin{array}{c}\text { Determinar la prevalencia } \\
\text { de caries dental, pérdida } \\
\text { dentaria y factores de } \\
\text { riesgo en la población } \\
\text { adulta chilena } \\
\end{array}$ & $\begin{array}{c}\text { Caries y } \\
\text { desdentamiento }\end{array}$ \\
\hline $\begin{array}{l}\text { Ramírez et } \\
\text { al, } 2015\end{array}$ & Nacional & $\begin{array}{l}\text { Estudio } \\
\text { ecológico }\end{array}$ & $\begin{array}{c}\text { Defunciones } \\
\text { obtenidas del } \\
\text { Departamento } \\
\text { de Estadística } \\
\text { e Información } \\
\text { de Salud del } \\
\text { Ministerio de } \\
\text { Salud de Chile } \\
\end{array}$ & $\begin{array}{l}\text { Universida de Los } \\
\text { Andes }\end{array}$ & 2002- 2010 & $\begin{array}{l}\text { Artículo de } \\
\text { Investigación }\end{array}$ & $\begin{array}{l}\text { Describir la mortalidad } \\
\text { por cáncer oral y faríngeo } \\
\text { en Chile entre los años } \\
\text { 2002- } 2010\end{array}$ & $\begin{array}{l}\text { Mortalidad por cáncer } \\
\text { oral y faríngeo }\end{array}$ \\
\hline $\begin{array}{l}\text { MINSAL, } \\
\text { PUC. } 2019\end{array}$ & Nacional & $\begin{array}{l}\text { Encuesta } \\
\text { poblacional de } \\
\text { tipo transversal. } \\
\text { Examen dental } \\
\text { realizado por } \\
\text { enfermera }\end{array}$ & $\begin{array}{l}\text { Personas } \geq 15 \\
\text { años }(n=6233)\end{array}$ & MINSAL & 2016- 2017 & Informe técnico & $\begin{array}{l}\text { Estimar la prevalencia } \\
\text { de problemas de } \\
\text { salud prioritarios y sus } \\
\text { determinantes en una } \\
\text { muestra nacional de } \\
\text { hogares, representativa } \\
\text { de la población general } \\
\text { chilena no } \\
\text { institucionalizada de } 15 \text { y } \\
\text { más años. }\end{array}$ & $\begin{array}{l}\text { Caries dental y } \\
\text { desdentamiento }\end{array}$ \\
\hline
\end{tabular}


Tabla 2. Estudios poblacionales de diagnóstico de salud oral de alcance Regional.

\begin{tabular}{|c|c|c|c|c|c|c|c|c|}
\hline Autor & Alcance & Tipo de estudio & Población objetivo & Financiamiento & $\begin{array}{c}\text { Año de } \\
\text { desarrollo }\end{array}$ & $\begin{array}{c}\text { Tipo de } \\
\text { publicación }\end{array}$ & Objetivo & $\begin{array}{c}\text { Evento de salud } \\
\text { oral }\end{array}$ \\
\hline $\begin{array}{l}\text { Gamonal et } \\
\text { al, } 1998\end{array}$ & $\begin{array}{c}\text { Región } \\
\text { Metropolitana }\end{array}$ & $\begin{array}{l}\text { Estudio de } \\
\text { Prevalencia }\end{array}$ & $\begin{array}{c}\text { Adultos de } 35-44 \\
(\mathrm{n}=879) \text { y } 65-74 \text { años } \\
(\mathrm{n}=217)\end{array}$ & Fondecyt, Conicyt & No indica & $\begin{array}{l}\text { Artículo de } \\
\text { Investigación }\end{array}$ & $\begin{array}{c}\text { Determinar la necesidad de } \\
\text { tratamiento en adultos de la Región } \\
\text { Metropolinana de Chile, utilizando } \\
\text { CPITN }\end{array}$ & $\begin{array}{l}\text { Enfermeddad } \\
\text { periodontal, } \\
\text { desdentamiento }\end{array}$ \\
\hline $\begin{array}{l}\text { Espinoza et } \\
\text { al, } 2003\end{array}$ & $\begin{array}{c}\text { Región } \\
\text { Metropolitana }\end{array}$ & $\begin{array}{l}\text { Estudio de } \\
\text { Prevalencia }\end{array}$ & $\begin{array}{c}\text { Adultos } \geq 65 \text { años }(n= \\
889)\end{array}$ & $\begin{array}{l}\text { Universidad de } \\
\text { Chile }\end{array}$ & No indica & $\begin{array}{l}\text { Artículo de } \\
\text { Investigación }\end{array}$ & $\begin{array}{c}\text { Determinar la prevalencia de } \\
\text { lesiones de mucosa oral y asociarlas } \\
\text { a diversos factores en población } \\
\text { mayor chilena } \\
\text { prioritarias y su variabilidad, en } \\
\text { población adulta chilena }\end{array}$ & $\begin{array}{l}\text { Lesiones de } \\
\text { mucosa oral }\end{array}$ \\
\hline $\begin{array}{l}\text { Mariño et al, } \\
2011\end{array}$ & $\begin{array}{l}\text { Región de } \\
\text { Valparaíso }\end{array}$ & $\begin{array}{l}\text { Estudio de } \\
\text { prevalencia }\end{array}$ & $\begin{array}{c}\text { Adultos } \geq 60 \text { años }(n= \\
354)\end{array}$ & FONIS, Conicyt & 2006-2007 & $\begin{array}{l}\text { Artículo de } \\
\text { Investigación }\end{array}$ & $\begin{array}{c}\text { Determinar el estado de salud oral } \\
\text { y la necesidad de tratamiento en } \\
\text { adultos mayores de la Región de } \\
\text { Valparaíso }\end{array}$ & $\begin{array}{l}\text { Enfermedad } \\
\text { periodontal, } \\
\text { desdentamiento, } \\
\text { caries dental } \\
\text { y lesiones de } \\
\text { mucosa oral }\end{array}$ \\
\hline $\begin{array}{l}\text { Mariño et al, } \\
2014\end{array}$ & $\begin{array}{l}\text { Región del } \\
\text { Maule }\end{array}$ & $\begin{array}{l}\text { Estudio de } \\
\text { prevalencia }\end{array}$ & $\begin{array}{l}\text { Adultos } 65-74 \text { años } \\
\qquad(n=438)\end{array}$ & $\begin{array}{l}\text { Universidad de } \\
\text { Talca }\end{array}$ & 2011 & $\begin{array}{c}\text { Artículo de } \\
\text { Investigación }\end{array}$ & $\begin{array}{c}\text { Determinar el estado de salud oral } \\
\text { y necesidad de tratamiento en } \\
\text { población de adultos mayores de la } \\
\text { Región del Maule, Chile }\end{array}$ & $\begin{array}{c}\text { Enfermedad } \\
\text { periodontal, } \\
\text { caries dental, } \\
\text { desdentamiento }\end{array}$ \\
\hline $\begin{array}{l}\text { Mariño et al, } \\
2015\end{array}$ & $\begin{array}{l}\text { Región del } \\
\text { Maule }\end{array}$ & $\begin{array}{l}\text { Estudio de } \\
\text { prevalencia }\end{array}$ & $\begin{array}{l}\text { Adultos } 65-74 \text { años } \\
\qquad(n=328)\end{array}$ & $\begin{array}{l}\text { Universidad de } \\
\text { Talca }\end{array}$ & 2011 & $\begin{array}{l}\text { Artículo de } \\
\text { Investigación }\end{array}$ & $\begin{array}{c}\text { Determinar la prevalencia de } \\
\text { caries radicular y necesidades de } \\
\text { tratamiento en población ambulatoria } \\
\text { de adultos mayores de la región del } \\
\text { Maule, Chile }\end{array}$ & Caries dental \\
\hline $\begin{array}{l}\text { Giacaman et } \\
\text { al, } 2016\end{array}$ & $\begin{array}{l}\text { Región del } \\
\text { Maule }\end{array}$ & $\begin{array}{l}\text { Estudio de } \\
\text { prevalencia }\end{array}$ & $\begin{array}{c}6 \text { años }(n=485) \\
12 \text { años }(n=552) \\
15 \text { años }(n=486) \\
\text { 35-44 años }(n=453) \\
65-74 \text { años }(n=438)\end{array}$ & $\begin{array}{l}\text { IADR, Universidad } \\
\text { de Talca }\end{array}$ & 2011 & $\begin{array}{c}\text { Artículo de } \\
\text { Investigación }\end{array}$ & $\begin{array}{c}\text { Determinar estado periodontal de } \\
\text { la población de } 6,12,15,33-44 \text { y } \\
65-74 \text { años de la Región del Maule, } \\
\text { Chile }\end{array}$ & $\begin{array}{l}\text { Enfermedad } \\
\text { periodontal }\end{array}$ \\
\hline $\begin{array}{l}\text { Mariño et al, } \\
2017\end{array}$ & $\begin{array}{l}\text { Región del } \\
\text { Maule }\end{array}$ & $\begin{array}{l}\text { Estudio de } \\
\text { Prevalencia }\end{array}$ & $\begin{array}{l}\text { Adultos } 65-74 \text { años } \\
\qquad(n=438)\end{array}$ & $\begin{array}{l}\text { Universidad de } \\
\text { Talca }\end{array}$ & 2011 & $\begin{array}{l}\text { Artículo de } \\
\text { Investigación }\end{array}$ & $\begin{array}{c}\text { Describir el uso de servicios de } \\
\text { salud oral en la población de adultos } \\
\text { mayores de la Región del Maule, } \\
\text { Chile }\end{array}$ & Desdentamiento \\
\hline
\end{tabular}

Tabla 3. Estudios poblacionales de diagnóstico de Caries Dental de alcance Nacional y Regional, financiados y no financiados.

\begin{tabular}{|c|c|c|c|c|c|c|c|c|c|c|c|c|c|}
\hline \multirow{3}{*}{ Autor } & \multirow{3}{*}{ Alcance } & \multicolumn{12}{|c|}{ Resultados } \\
\hline & & \multicolumn{3}{|c|}{ Prevalencia Caries Cavitada } & \multirow{2}{*}{$\begin{array}{l}\text { Promedio } \\
\text { dientes } \\
\text { cariados }\end{array}$} & \multicolumn{6}{|c|}{ COPD } & \multicolumn{2}{|c|}{ Caries Radicular } \\
\hline & & Total & Edad & Género & & Total & Edad & Género & Cariado & Obturado & Perdido & Índice & Historia \\
\hline $\begin{array}{l}\text { MINSAL, } \\
\text { PUC. } \\
2003\end{array}$ & $\begin{array}{l}\text { Nacional (personas } \\
\geq 17 \text { años) }\end{array}$ & $70.0 \%$ & $\begin{array}{c}\text { 17-24 años: } 56.4 \% \\
25-44 \text { años: } 75.5 \% \\
45-64 \text { años: } 67.1 \% \\
\geq 65 \text { años: } 44.7 \%\end{array}$ & $\begin{array}{l}\text { Hombre: } \\
71.2 \% \\
\text { Mujer: } \\
68.8 \%\end{array}$ & 2.5 & NR & NR & NR & NR & NR & NR & NR & NR \\
\hline \multirow{2}{*}{$\begin{array}{l}\text { Urzúa et } \\
\text { al, } 2012\end{array}$} & \multirow{2}{*}{$\begin{array}{l}\text { Nacional (adultos 35- } \\
44 \text { y } 65-74 \text { años) }\end{array}$} & \multirow{2}{*}{ NR } & \multirow{2}{*}{ NR } & \multirow[b]{2}{*}{ NR } & \multirow[b]{2}{*}{ NR } & 15.06 & $\begin{array}{l}\text { 35-44 años: } \\
15.06\end{array}$ & $\begin{array}{c}\text { Hombre: } \\
14.1 \\
\text { Mujer: } \\
15.86 \\
\end{array}$ & 4.2 & 4.9 & 5.9 & \multirow[b]{2}{*}{ NR } & \multirow[b]{2}{*}{ NR } \\
\hline & & & & & & 21,57 & $\begin{array}{l}\text { 65-74 años: } \\
21.57\end{array}$ & $\begin{array}{c}\text { Hombre: } \\
14.1 \\
\text { Mujer: } \\
15.86 \\
\end{array}$ & 1.44 & 2.66 & 17.46 & & \\
\hline $\begin{array}{l}\text { MINSAL, } \\
\text { PUC. } \\
2019\end{array}$ & $\begin{array}{l}\text { Nacional (personas } \\
\geq 15 \text { años) }\end{array}$ & $54.6 \%$ & $\begin{array}{c}\text { 15-24 años: } 40.9 \% \\
\text { 25-44 años: } 57.1 \% \\
45-64 \text { años: } 59.6 \% \\
\geq 65 \text { años: } 57.2 \%\end{array}$ & $\begin{array}{l}\text { Hombre: } \\
58.4 \% \\
\text { Mujer: } \\
50.6 \%\end{array}$ & NR & NR & NR & NR & NR & NR & NR & NR & NR \\
\hline $\begin{array}{l}\text { Mariño et } \\
\text { al, } 2011\end{array}$ & $\begin{array}{l}\text { Región de Valparaíso } \\
\text { (adultos } \geq 60 \text { años) }\end{array}$ & NR & NR & NR & NR & 21.6 & NR & $\begin{array}{c}\text { Hombre: } \\
22.6 \\
\text { Mujer: } \\
20.6\end{array}$ & \multicolumn{2}{|c|}{4.7} & 17.9 & NR & NR \\
\hline $\begin{array}{l}\text { Mariño et } \\
\text { al, } 2014\end{array}$ & $\begin{array}{l}\text { Región del Maule } \\
\text { (adultos 65-74 años) }\end{array}$ & NR & NR & NR & NR & NR & $\begin{array}{c}65-69 \text { años: } \\
24.4 \\
70-74 \text { años: } \\
26.9 \\
\end{array}$ & $\begin{array}{c}\text { Hombre: } \\
24.4 \\
\text { Mujer: } \\
26.2\end{array}$ & \multicolumn{2}{|c|}{3.3} & 22.4 & NR & NR \\
\hline $\begin{array}{l}\text { Mariño et } \\
\text { al, } 2015\end{array}$ & $\begin{array}{c}\text { Región del Maule } \\
\text { (adultos 65-74 años) }\end{array}$ & NR & NR & NR & NR & NR & NR & NR & NR & NR & NR & $8.23 \%$ & 0.76 \\
\hline
\end{tabular}

COPD: Dientes cariados, obturados, perdidos por caries

NR: No reporta

indice de caries radicular:

inserción clínica $\geq 7 \mathrm{~mm}$., fueron presentar una mayor edad, sexo masculino, bajo nivel educacional ( $\leq 12$ años) y ser fumador(13).

A nivel regional, se identificaron 4 estudios $^{(15,17,18,20)}$, donde en la mayoría de ellos, se analizó como variable primaria el índice periodontal comunitario o índice de necesidad de tratamiento de la comunidad (CPITN). En la Región Metropolitana, el código 4 del CPITN fue mayor en los sujetos de 65 - 74 años, en los niveles socioeconómicos medios y bajos, en los hombres y en aquellos con $\leq 8$ años de educación. La necesidad de un tratamiento periodontal complejo para resolver código 3 y 4, en la Región Metropolitana, fue de un $40.66 \%$ en los sujetos de 35 a 44 años, aumentando significativamente a $71.43 \%$ en los sujetos de 65 a 74 años. Para ello, se requerían un total de 1.350 .000 horas para resolver las necesidades de tratamiento de ambos grupos etareos ${ }^{(15)}$. En la Región de Valparaíso, se reportó que la necesidad de tratamiento periodontal 
complejo en sujetos $\geq 60$ años, fue de un $43.0 \%$, siendo mayor en hombres que en mujeres ${ }^{(17)}$. En la Región del Maule, en los sujetos de 35 a 44 años, la necesidad de tratamiento periodontal complejo fue de un $19.7 \%{ }^{(20)}$, y en los sujetos de 65 a 74 años, de un $28.8 \%^{(20)}$ a $30.1 \%^{(18)}$. CPITN 3 y 4 fue mayor en los sujetos con $\leq 8$ años de educación y aquellos que vivían en áreas rurales ${ }^{(18)}$. En el grupo de 65-74 años, los sujetos con pérdida de inserción clínica $\geq 4 \mathrm{~mm}$., correspondían a un $87.9 \%$, y a un $47.7 \%$ para la pérdida de inserción clínica $\geq 6 \mathrm{~mm}$.

Estudios poblacionales de diagnóstico de Desdentamiento (Tabla

A nivel nacional, se identificaron 4 estudios $^{(9,10,13,14)}$, donde 2 analizaban la misma muestra de sujetos ${ }^{(13,14)}$. La prevalencia de dentición no funcional ( $<20$ dientes en boca) presentó una prevalencia de un $32.3 \%$ en los sujetos $\geq 17$ años el año $2003^{(9)}$, disminuyendo significativamente a un $27.0 \%$ en los sujetos $\geq 15$ años en los años 2016-2017(10). En la ENS 2016- 2017, la prevalencia de dentición no funcional fue mayor en mujeres, en sujetos de mayor edad y con $<8$ años de educación ${ }^{(10)}$. En el grupo de 35- 44 años, la presencia de $\leq 20$ dientes se asociaba con bajo nivel educacional y de ingresos y depresión autoreportada ${ }^{(14)}$.

La media de pérdida dentaria fue de 5.9 a 6.5 en los sujetos de 35 a 44 $\operatorname{años}^{(13,14)}$ y de 15.8 a 17.46 dientes en los sujetos de 65 a 74 años ${ }^{(13,14)}$. El grupo de 35- 44 años, presentó una menor pérdida dentaria que el grupo de $65-74$ años $^{(14)}$. La prevalencia de desdentados totales fue de un 0.28 a un $0.7 \%{ }^{(12,15,16)}$ en los sujetos de 35 a 44 años, y de un $17.6 \%$ a un $20.6 \%$ en los sujetos de 65 a 74 años ${ }^{(10,13,14)}$. El uso de prótesis disminuyó del $25.0 \%$ en el año 2003(9), a un $22.4 \%$ en los años $2016-2017^{(10)}$. En la ENS 2016-2017, el uso de prótesis fue mayor en las mujeres, en los grupos de mayor edad y en los sujetos con $<8$ años de estudio(10).

A nivel regional, se identificaron 4 estudios ${ }^{(15,17,18,21)}$. En la Región Metropolitana, la media de pérdida dentaria en población de 35 a 44 años fue de 6.62 dientes y en la población de 65 a 74 años, 12.29 dientes, siendo mayor en el grupo de 65 a 74 años y en el nivel socioeconómico bajo. La principal causa de pérdida dentaria fue la caries dental, seguida de periodontitis ${ }^{(15)}$. En la Región de Valparaíso, en los sujetos $\geq 60$ años, la media de pérdida dentaria fue de un 17.9 dientes, donde la prevalencia de desdentados totales fue de un $20.1 \%$, y la necesidad protésica ascendía a un $70.3 \%{ }^{(17)}$. En la Región del Maule, en los adultos de 65 a 74 años, la pérdida dentaria fue de un 22.4 dientes ${ }^{(18)}$, siendo mayor en los sujetos de más edad, menor educación ( $\leq 8$ años $)^{(18)}$ y los que no utilizaron el servicio dental en los últimos 12 meses ${ }^{(21)}$. La prevalencia de desdentados totales fue de un $25.1 \%$, y la necesidad prótesica estaba presente en el $72.0 \%$ de la población ${ }^{(18)}$.

Estudios poblacionales de diagnóstico de Lesiones de Mucosa Oral y Cáncer Oral y Faríngeo (Tabla 6)

A nivel nacional, se identificaron 2 estudios, que correspondieron a un análisis secundario de datos de mortalidad por cáncer oral y faríngeo ${ }^{(11,12)}$. La tasa bruta de mortalidad por cáncer oral y orofaríngeo fue de 0.9 a 1.25 por cada 100.000 habitantes, donde la mortalidad por cáncer de lengua fue la más prevalente.

A nivel regional, se identificaron 2 estudios ${ }^{(16,17)}$. En la Región Metropolitana, en los adultos $\geq 65$ años, la prevalencia de lesiones de mucosa oral fue de un $53.0 \%$, donde la patología más frecuente fue la estomatitis subprotésica (22.3\%). Las leucoplasias presentaron una prevalencia de un $1.7 \%$ y el liquen plano, de un $2.1 \%$. El uso de prótesis aumentaba el riesgo de cualquier lesión de mucosa oral en población adulta mayor. Los indicadores de riesgo que aumentaron el riesgo de estomatitis subprotésica fueron el género femenino, prótesis con placa bacteriana adherida y dormir con prótesis. Los indicadores de riesgo para liquen plano fue ser del género femenino, y para leucoplasia, fue ser fumador ${ }^{(16)}$. En la Región de Valparaíso, en los adultos $\geq 60$ años, la prevalencia de lesiones de mucosa oral fue de un $34.2 \%$, con necesidad de atención inmediata por especialista producto de presentar lesiones potencialmente malignas de un $1.7 \%{ }^{(17)}$

En la sinopsis realizada en este estudio, se reporta que la prevalencia de enfermedades orales es alta en adultos y personas mayores chilenas, donde la población más afectada es la de menores recursos económicos y la de mayor edad.

Si bien la prevalencia de caries cavitadas en las personas $\geq 17$ años disminuyó considerablemente de un $66.3 \%$ a un $54.6 \%$ entre los años $2003^{(9)}$ al 2016- $2017^{(10)}$, sigue siendo mayor a la prevalencia reportada a nivel mundial, la cual cifra la frecuencia de caries cavitadas en dentición permanente en un $29.4 \%{ }^{(2)}$. Además, esta condición aumentó en los sujetos $\geq 65$ años, pudiendo deberse al aumento de la retención de dientes en este grupo de edad, sumado a una mayor incidencia de caries radiculares. Además, el número de dientes afectados por caries (historia de caries COPD) en los grupos etarios de 35- 44 años a 65- 74 años fue de 15.06 y $21.57^{(14)}$, respectivamente, lo que representa más de un $50 \%$ de los dientes afectados por caries cavitadas en la edad adulta y sobre un $75 \%$ de los dientes presentes en boca en las personas mayores.

La prevalencia de dentición funcional fue baja, aunque aumentó significativamente entre los años $2003^{(9)}$ y $2016-2017^{(10)}$, pero donde los adultos $\geq 65$ años, presentaron una prevalencia de dentición funcional de solo un $22.5 \%$. Los hallazgos del estudio con respecto a la edad confirman lo que otros trabajos han demostrado con anterioridad; a medida que aumenta la edad disminuye el número de dientes presentes en la boca, observando en las personas mayores una prevalencia de desdentamiento total a nivel país de $58.8 \%{ }^{(10)}$, superando ampliamente la media nacional de $5.5 \%$, y la mundial, de un $3.3 \%^{(2)}$. Lo aumentado de estas cifras se explica en la pérdida dentaria parcial de la adultez, donde el número de dientes perdidos entre los 35- 44 años ya es de 6.6 dientes ${ }^{(13)}$. Esta situación refleja un escenario preocupante, debido a que nos encontramos en una transición demográfica, donde el número de personas mayores irá aumentando. Sumado a esto, uno de los indicadores recomendados por la Organización Mundial de la Salud (OMS) para medir la salud en los adultos mayores es la evaluación de la mantención o pérdida de funcionalidad(24), debido al impacto que tiene en su estado estado de salud y calidad de vida. Por lo tanto, uno de los objetivos de las políticas públicas para personas adultas debe orientarse a aumentar los años libres de discapacidad

Tabla 4. Estudios poblacionales de diagnóstico de Enfermedad Periodontal de alcance Nacional y Regional, financiados o no financiados

\begin{tabular}{|c|c|c|c|c|c|c|c|c|c|c|c|c|c|c|c|c|c|c|}
\hline \multirow[b]{3}{*}{ Autor } & \multirow[b]{3}{*}{ Alcance } & \multirow[b]{3}{*}{ Edad } & \multicolumn{16}{|c|}{ Resultados } \\
\hline & & & \multicolumn{7}{|c|}{ Pérdida de inserción clínica } & \multicolumn{5}{|c|}{ CPITN (códigos) } & \multicolumn{4}{|c|}{ Necesidad de tratamiento } \\
\hline & & & $\leq 3 \mathrm{~mm}$ & $\geq 4 \mathrm{~mm}$ & $\geq 5 \mathrm{~mm}$ & $\geq 6 \mathrm{~mm}$ & $\geq 7 \mathrm{~mm}$ & $\geq 8 \mathrm{~mm}$ & $\geq 9 \mathrm{~mm}$ & 0 & 1 & 2 & 3 & 4 & $\begin{array}{c}\text { Sin } \\
\text { necesidad }\end{array}$ & IHO & Destartraje & \begin{tabular}{|c|}
$\begin{array}{c}\text { Tratamiento } \\
\text { periodontal } \\
\text { complejo }\end{array}$ \\
\end{tabular} \\
\hline $\begin{array}{l}\text { Gamonal } \\
\text { et al, } 2010\end{array}$ & $\begin{array}{c}\text { Nacional } \\
\text { (adultos } 35 \text { - } \\
44 \text { y } 65-74 \\
\text { años) }\end{array}$ & $\begin{array}{c}35-44 \\
\text { años } \\
65-74 \\
\text { años }\end{array}$ & $\begin{array}{l}6.6 \% \\
2.4 \%\end{array}$ & $\begin{array}{l}93.4 \% \\
97.5 \%\end{array}$ & $\begin{array}{l}77.5 \% \\
92.7 \%\end{array}$ & $\begin{array}{l}58.3 \% \\
81.4 \%\end{array}$ & $\begin{array}{l}38.6 \% \\
69.3 \%\end{array}$ & NR & NR & NR & NR & NR & NR & NR & NR & NR & NR & NR \\
\hline $\begin{array}{r}\text { Gamonal } \\
\text { et al, } 1998\end{array}$ & $\begin{array}{c}\text { Región } \\
\text { Metropolitana } \\
\text { (adultos } 35 \text { - } \\
44 \text { y } 65 \text { - } 74 \\
\text { años) }\end{array}$ & $\begin{array}{c}\text { 35-44 } \\
\text { años } \\
65-74 \\
\text { años }\end{array}$ & NR & NR & NR & NR & NR & NR & NR & $\begin{array}{l}1.3 \% \\
0.0 \%\end{array}$ & $\begin{array}{l}3.0 \% \\
0.0 \%\end{array}$ & $\begin{array}{l}4.9 \% \\
0.0 \%\end{array}$ & $\begin{array}{l}50.2 \% \\
54.8 \%\end{array}$ & $\begin{array}{l}40.7 \% \\
36.6 \%\end{array}$ & $\begin{array}{l}1.3 \% \\
0.0 \%\end{array}$ & $\begin{array}{c}98.8 \% \\
100 \%\end{array}$ & $\begin{array}{l}98.8 \% \\
100 \%\end{array}$ & $\begin{array}{l}40.7 \% \\
71.4 \%\end{array}$ \\
\hline $\begin{array}{c}\text { Mariño et } \\
\text { al, } 2011\end{array}$ & $\begin{array}{c}\text { Región de } \\
\text { Valparaíso } \\
\text { (adultos } \geq 60 \\
\text { años) }\end{array}$ & $\begin{array}{l}\geq 60 \\
\text { años }\end{array}$ & NR & NR & NR & NR & NR & NR & NR & $3.3 \%$ & $11.4 \%$ & $42.3 \%$ & 43. & $0 \%$ & NR & NR & NR & $43.0 \%$ \\
\hline $\begin{array}{l}\text { Mariño et } \\
\text { al, } 2014\end{array}$ & $\begin{array}{c}\text { Región } \\
\text { del Maule } \\
\text { (adultos } 65 \text { - } \\
74 \text { años) }\end{array}$ & $\begin{array}{c}65-74 \\
\text { años }\end{array}$ & $12.1 \%$ & $87.9 \%$ & NR & $47.7 \%$ & NR & NR & $14.5 \%$ & $0.0 \%$ & $1.5 \%$ & $68.4 \%$ & $25.5 \%$ & $4.6 \%$ & NR & NR & NR & $30.1 \%$ \\
\hline $\begin{array}{l}\text { Giacaman } \\
\text { et al, } 2016\end{array}$ & \begin{tabular}{|c|} 
Región del \\
Maule $(6,12$, \\
$15,35-44,65$ \\
-74 años)
\end{tabular} & $\begin{array}{c}\text { 35-44 } \\
\text { años } \\
65-74 \\
\text { años }\end{array}$ & NR & NR & NR & NR & NR & NR & NR & $\begin{array}{l}0.2 \% \\
0.0 \%\end{array}$ & $\begin{array}{l}2.9 \% \\
1.5 \%\end{array}$ & $\begin{array}{l}77.2 \% \\
65.8 \%\end{array}$ & $\begin{array}{l}17.2 \% \\
25.0 \%\end{array}$ & $\begin{array}{l}2.5 \% \\
3.8 \%\end{array}$ & NR & NR & NR & $\begin{array}{l}19.70 \% \\
28.8 \%\end{array}$ \\
\hline
\end{tabular}

CPITN/ índice periodontal comunitario: Código 0: Salud; Código 1: Sangrado al sondaje: Código 2: Cálculo; Código 3: Profundidad al sondaje de 4-5mm; Código 4: Profundidad al sondaje >5mm IHO: Instrucción de higiene oral

NR: No reporta 
Tabla 5. Estudios poblacionales de diagnóstico de Desdentamiento de alcance Nacional y Regional, financiados o no financiados.

\begin{tabular}{|c|c|c|c|c|c|c|c|c|c|c|c|c|c|c|c|c|c|c|c|c|c|}
\hline \multirow[t]{2}{*}{ Autor } & \multirow[t]{2}{*}{ Alcance } & \multirow{2}{*}{$\begin{array}{l}\text { Número } \\
\text { de dientes } \\
\text { presentes }\end{array}$} & \multicolumn{3}{|c|}{$\begin{array}{l}\text { Número de dientes } \\
\text { perdidos }\end{array}$} & \multicolumn{3}{|c|}{ Dentición completa } & \multicolumn{3}{|c|}{ Desdentamiento parcial } & \multicolumn{3}{|c|}{ Dentición no funcional } & \multicolumn{3}{|c|}{ Desdentamiento total } & \multicolumn{3}{|c|}{ Uso de prótesis } & \multirow{2}{*}{$\begin{array}{c}\text { Nece- } \\
\text { sidad } \\
\text { protésica }\end{array}$} \\
\hline & & & Total & Edad & Género & Total & Edad & Género & Total & Edad & Género & Total & Edad & Género & Total & Edad & Género & Total & Edad & Género & \\
\hline $\begin{array}{l}\text { MINSAL, } \\
\text { PUC. } \\
2003\end{array}$ & $\begin{array}{l}\text { Nacional } \\
\text { (personas } \\
\geq 17 \text { años) }\end{array}$ & 21.43 & NR & NR & NR & $27.8 \%$ & \begin{tabular}{|c|}
$17-24$ \\
a: \\
$61.8 \%$ \\
$25-$ \\
$44 a:$ \\
$32.0 \%$ \\
$45-$ \\
$64 a:$ \\
$7.4 \%$ \\
$\geq 65 a:$ \\
$0.7 \%$ \\
\end{tabular} & $\begin{array}{l}\text { Hombre: } \\
29.2 \% \\
\text { Mujer: } \\
27.8 \%\end{array}$ & NR & NR & NR & $29.7 \%$ & NR & $\begin{array}{c}\text { Hombre: } \\
25.0 \% \\
\text { Mujer: } \\
34.2 \%\end{array}$ & $5.5 \%$ & $\begin{array}{c}17- \\
24 \mathrm{a}: \\
0.0 \% \\
25- \\
44 \mathrm{a}: \\
0.2 \% \\
45- \\
64 \mathrm{a}: \\
6.7 \% \\
\geq 65 \mathrm{a}: \\
33.4 \% \\
\end{array}$ & $\begin{array}{c}\text { Hombre: } \\
3.3 \% \\
\text { Mujer: } \\
7.7 \%\end{array}$ & $25.0 \%$ & NR & $\begin{array}{c}\text { Hombre: } \\
19 \% \\
\text { Mujer: } \\
30 \%\end{array}$ & NR \\
\hline $\begin{array}{l}\text { Gamonal } \\
\text { et al, } \\
2010\end{array}$ & \begin{tabular}{|l} 
Nacional \\
(adultos \\
$35-44$ \\
y 65-74 \\
años)
\end{tabular} & NR & 6.5 & NR & NR & NR & NR & NR & NR & NR & NR & NR & NR & NR & $6.7 \%$ & \begin{tabular}{|c|}
$35-$ \\
$44 \mathrm{a}:$ \\
$0.7 \%$ \\
$65-$ \\
$74 \mathrm{a}:$ \\
$20.6 \%$ \\
\end{tabular} & NR & NR & NR & NR & NR \\
\hline Urzúa et & $\begin{array}{l}\text { Nacional } \\
\text { (adultos } \\
35-44\end{array}$ & $\begin{array}{l}<15: 4.2 \% \\
<21: 9.8 \% \\
\geq 21: 85.6 \% \\
\end{array}$ & 5.9 & NR & NR & NR & NR & NR & NR & NR & NR & NR & NR & NR & NR & $\begin{array}{c}35- \\
44 a: \\
0.28 \%\end{array}$ & NR & NR & NR & NR & NR \\
\hline al, 2012 & $\begin{array}{l}35-44 \\
\text { y 65-74 } \\
\text { años) }\end{array}$ & \begin{tabular}{|l|}
$<15: 43.6 \%$ \\
$<21: 21.1 \%$ \\
$\geq 21: 23.9 \%$ \\
\end{tabular} & 17.5 & NR & NR & NR & NR & NR & NR & NR & NR & NR & NR & NR & NR & $\begin{array}{c}65- \\
74 a: \\
11.4 \%\end{array}$ & NR & NR & NR & NR & NR \\
\hline $\begin{array}{l}\text { MINSAL, } \\
\text { PUC. } \\
2019\end{array}$ & $\mid \begin{array}{l}\text { Nacional } \\
\text { (personas } \\
\geq 15 \text { años) }\end{array}$ & NR & NR & NR & NR & NR & $\begin{array}{c}15- \\
24 a: \\
68.1 \% \\
25- \\
34 a: \\
59.6 \% \\
35- \\
44 a: \\
30.6 \% \\
45- \\
54 a: \\
13.0 \% \\
55- \\
64 a: \\
7.8 \% \\
65- \\
74 a: \\
2.6 \% \\
\geq 75 a: \\
0.9 \% \\
\end{array}$ & NR & NR & $\begin{array}{c}15- \\
24 a: \\
31.9 \% \\
25- \\
34 a: \\
40.4 \% \\
35- \\
44 a: \\
69.0 \% \\
45- \\
54 a: \\
85.6 \% \\
55- \\
64 a: \\
86.0 \% \\
65- \\
74 a: \\
79.8 \% \\
\geq 75 a: \\
57.8 \% \\
\end{array}$ & NR & $24.8 \%$ & $\begin{array}{c}15- \\
24 \mathrm{a}: \\
0.4 \% \\
25- \\
44 \mathrm{a}: \\
4.7 \% \\
45- \\
64 \mathrm{a}: \\
41.5 \% \\
\geq 65 \mathrm{a}: \\
77.5 \%\end{array}$ & $\begin{array}{c}\text { Hombre: } \\
20.9 \% \\
\text { Mujer: } \\
29.1 \%\end{array}$ & NR & $\begin{array}{c}15- \\
24 a: \\
0.0 \% \\
25- \\
34 a: \\
0.0 \% \\
35- \\
44 a: \\
0.4 \% \\
45- \\
54 a: \\
1.4 \% \\
55- \\
64 a: \\
6.2 \% \\
65- \\
74 a: \\
17.6 \% \\
\geq 75 a: \\
41.2 \% \\
\end{array}$ & NR & $22.4 \%$ & $\begin{array}{c}15- \\
24 \mathrm{a}: \\
0.2 \% \\
25- \\
44 \mathrm{a}: \\
6.4 \% \\
45- \\
64 \mathrm{a}: \\
36.8 \% \\
\geq 65 \mathrm{a}: \\
65.8 \%\end{array}$ & $\begin{array}{c}\text { Hombre: } \\
15.6 \% \\
\text { Mujer: } \\
29.1 \%\end{array}$ & NR \\
\hline $\begin{array}{l}\text { Gamonal } \\
\text { et al, } \\
1998\end{array}$ & \begin{tabular}{|l|} 
Región \\
Metro- \\
politana \\
(adultos \\
$35-44$ y \\
$65-74$ \\
años) \\
\end{tabular} & NR & NR & $\begin{array}{c}35- \\
44 a: \\
6.62 \\
65- \\
74 a: \\
12.29 \\
\end{array}$ & NR & NR & NR & NR & NR & NR & NR & NR & NR & NR & NR & NR & NR & NR & NR & NR & NR \\
\hline $\begin{array}{l}\text { Mariño et } \\
\text { al, } 2011\end{array}$ & $\begin{array}{l}\text { Región de } \\
\text { Valparaíso } \\
\text { (adultos } \\
\geq 60 \text { años) } \\
\end{array}$ & NR & 17.9 & & NR & NR & NR & NR & NR & NR & NR & NR & NR & NR & $21.0 \%$ & NR & NR & NR & NR & NR & $70.3 \%$ \\
\hline $\begin{array}{l}\text { Mariño et } \\
\text { al, } 2014\end{array}$ & \begin{tabular}{|l|} 
Región \\
del Maule \\
(adultos \\
$65-74$ \\
años) \\
\end{tabular} & \begin{tabular}{|c|}
$1-10:$ \\
$34.2 \%$ \\
$11-20:$ \\
$25.1 \%$ \\
$\geq 20: 15.6 \%$ \\
\end{tabular} & 22.4 & $\begin{array}{c}65- \\
69 a: \\
19.9 \\
70-74 \\
\text { a: } 24.5\end{array}$ & $\begin{array}{c}\text { Hombres: } \\
20.6 \\
\text { Mujeres: } \\
22.9\end{array}$ & NR & NR & NR & NR & NR & NR & NR & NR & NR & $25.1 \%$ & NR & NR & NR & NR & NR & $72.0 \%$ \\
\hline $\begin{array}{l}\text { Mariño et } \\
\text { al, } 2017\end{array}$ & \begin{tabular}{|l|} 
Región \\
del Maule \\
(adultos \\
$65-74$ \\
años) \\
\end{tabular} & 10.5 & 21.5 & NR & NR & NR & NR & NR & NR & NR & NR & NR & NR & NR & $24.0 \%$ & NR & NR & NR & NR & NR & NR \\
\hline
\end{tabular}

Dentición no funcional: $<20$ dientes; Dentición completa: $\geq 14$ dientes por maxilar

NR: No reporta

mediante la retención de dientes en salud(25).

Los cambios en las prevalencias de caries cavitadas y de dentición no funcional podrían explicarse por la incorporación de las Garantías Explícitas en Salud (GES) de "Atención odontológica integral adulto de 60 años" en el año 2007, y del GES de "Salud oral integral de la embarazada"(26) en el año 2010. Sumado a esto, el Programa Más Sonrisas para Chile ha mejorado el acceso a la atención odontológica de las mujeres mayores de 20 años, permitiéndoles recuperar su salud bucal cuando la han perdido y entregándoles las herramientas para cuidarla. El Programa de Mejoramiento del Acceso a la Atención Odontológica para los Jóvenes de Cuarto Año de Educación Media, de colegios municipales y particulares subvencionados, y Atención de Morbilidad Odontológica de los adultos, han acercado la atención a las personas que por sus actividades académicas o laborales tienen dificultad para acceder a los lugares y en los horarios habituales de atención clínica ${ }^{(27)}$.

A nivel periodontal existe un único estudio nacional realizado el año 2010. La pérdida de inserción clínica $\geq 6 \mathrm{~mm}$., en los sujetos de 35 a 44 años fue de un $58.3 \%$, y de 65 a 74 años, de un $81.4 \%{ }^{(13)}$. Es mayor a los reportado a nivel mundial como prevalencia de "periodontitis severa", la que es definida como la presencia de pérdida de inserción clínica $\geq 6 \mathrm{~mm}$., o CPITN código 4. Ésta ha aumentado a un $9.8 \%{ }^{(2)}$ el año 2017 , habiéndose mantenido en un $7.4 \%$ entre los años 1990- 2015(1). Los estudios regionales de prevalencia de enfermedad periodontal, han utilizado como variable primaria el CPITN. Sin embargo, esto trae como consecuencia lo que ya se ha descrito a nivel de la epidemiología de las enfermedades periodontales a nivel mundial: la ausencia de uniformidad de la definición de caso, lo 
Tabla 6. Estudios poblacionales de diagnóstico de Lesiones de Mucosa Oral y Cáncer Oral y Orofaríngeo de alcance Nacional y Regional, financiados o no financiados

\begin{tabular}{|c|c|c|c|c|c|c|c|c|}
\hline \multirow[b]{3}{*}{ Autor } & \multirow[b]{3}{*}{ Alcance } & \multicolumn{7}{|c|}{ Resultados } \\
\hline & & \multicolumn{4}{|c|}{ Mortalidad por Cáncer Oral y Faríngeo } & \multicolumn{3}{|c|}{ Lesiones de mucosa oral } \\
\hline & & $\begin{array}{c}\text { Tasa bruta } \\
\text { (por } 100.000 \\
\text { habitantes) }\end{array}$ & Género & Edad & $\begin{array}{c}\text { Distribución anató- } \\
\text { mica }\end{array}$ & Prevalencia & Tipo de lesión & $\begin{array}{l}\text { Necesidad de } \\
\text { atención por } \\
\text { especialista }\end{array}$ \\
\hline $\begin{array}{l}\text { Riera y Martí- } \\
\text { nez, } 2005\end{array}$ & Nacional & $0.9-1.3$ & $\begin{array}{c}\text { Hombre: } \\
70.1 \% \\
\text { Mujer: } \\
29.9 \%\end{array}$ & NR & $\begin{array}{c}\text { Lengua: } 39.0 \% \\
\text { Glándulas salivales } \\
\text { mayores: } 30.0 \% \\
\text { Piso de boca: } 18.0 \% \\
\text { Labio: } 9.0 \% \\
\text { Encía: } 4.0 \% \\
\end{array}$ & NR & NR & NR \\
\hline $\begin{array}{l}\text { Ramírez et al, } \\
2015\end{array}$ & Nacional & $1.11-1.25$ & $\begin{array}{l}\text { Hombre: } \\
74.0 \% \\
\text { Mujer: } \\
26.0 \%\end{array}$ & $\begin{array}{c}\text { 10-14a: } 0.2 \% \\
\text { 15-24a: } 0.5 \% \\
\text { 25-34a: } 1.2 \% \\
\text { 35-44a: } 3.0 \% \\
\text { 45-54a: } 12.4 \% \\
\text { 55-64a: } 22.9 \% \\
\text { 65-74a: } 27.4 \% \\
\geq 75 a: 32.4 \%\end{array}$ & $\begin{array}{c}\text { Lengua: } 17.7 \% \\
\text { Orofaringe: } 17.1 \% \\
\text { Glándulas salivales } \\
\text { mayores: } 15.0 \% \\
\text { Hipofaringe: } 10.6 \% \\
\text { Piso de boca: } 9.4 \%\end{array}$ & NR & NR & NR \\
\hline $\begin{array}{l}\text { Espinoza et } \\
\text { al, } 2003\end{array}$ & $\begin{array}{c}\text { Región } \\
\text { Metropolitana } \\
\text { (adultos } \geq 65 \\
\text { años) }\end{array}$ & NR & NR & NR & NR & $53.0 \%$ & \begin{tabular}{|c|} 
Estomatitis subprotésica: $22.3 \%$ \\
Hiperplasia irritativa: $9.4 \%$ \\
Varicosidades de mucosa oral: $9.0 \%$ \\
Queratosis friccional: $6.0 \%$ \\
Lesiones únicas pigmentadas: $4.0 \%$ \\
Ulceraciones traumáticas: $3.5 \%$ \\
Queilitis angular: $2.9 \%$ \\
Lesiones múltiples pigmentadas: $2.8 \%$ \\
Hemangioma: $2.3 \%$ \\
Liquen plano: $2.1 \%$ \\
Leucoplasia: $1.7 \%$ \\
Estomatitis aftosa recurrente: $1.4 \%$ \\
Estomatitis nicotínica: $1.3 \%$ \\
Glositis romboidal media: $0.9 \%$ \\
Queilitis actínica: $0.9 \%$ \\
Granuloma piogénico: $0.7 \%$ \\
Papiloma escamoso oral: $0.6 \%$ \\
Mucocele: $0.2 \%$ \\
\end{tabular} & NR \\
\hline $\begin{array}{l}\text { Mariño et al, } \\
2011\end{array}$ & $\begin{array}{c}\text { Región de } \\
\text { Valparaíso } \\
\text { (adultos } \geq 60 \\
\text { años) }\end{array}$ & NR & NR & NR & NR & NR & NR & $1.7 \%$ \\
\hline
\end{tabular}

NR: No reporta

que imposibilita realizar comparaciones entre los distintos estudios epidemiológicos desarrollados ${ }^{(28,29)}$. Sumado a lo anterior, los índices utilizados consisten en un registro parcial de la boca, lo que subestima la magnitud y severidad de las enfermedades periodontales, no describe sus características al no considerar la pérdida de inserción clínica, si no que sólo identifica las necesidades de tratamiento de los sujetos, lo cual puede estar sobreestimado al aplicar un concepto jerárquico de progresión de las enfermedades periodontales ${ }^{(30)}$. Es imperativo que se desarrollen estudios utilizando la Clasificación de las Enfermedades Periodontales y Periimplantares del año $2017^{(31)}$, de modo que se permita la comparación con estudios internacionales, así como estudios de progresión de la enfermedad, sumado al tratamiento de esta patología, considerada un problema de salud pública ${ }^{(32)}$, así como una enfermedad crónica no transmisible ${ }^{(33)}$

Esta revisión muestra que la cantidad de artículos referentes al cáncer oral y faríngeo, así como de lesiones de mucosa oral, es bajo. La mortalidad por cáncer oral en Chile, según los distintos estudios, fluctúa entre $0.9-1.3$ por cada 100.000 habitantes ${ }^{(11,12)}$. El sexo más afectado fue el masculino, tal como en Estados Unidos, Europa y México ${ }^{(34)}$. Algunos de los principales factores de riesgo de cáncer oral son el tabaquismo y el consumo riesgososo de alcohol(35), aumentando su mortalidad. El tabaquismo también es un factor de riesgo para las leucoplasias, las cuales presentaron una prevalencia de un $1.7 \%$ en población adulta mayor en la Región Metropolitana ${ }^{(16)}$. Según la ENS 2016- 2017, la prevalencia de tabaquismo en Chile fue de un $33.3 \%$, y de alcohol, $11.7 \%{ }^{(36)}$. Debido a esto, se esperaría que se aumentaran los programas de salud enfocados a la prevención de ambos factores de riesgo. El control del cáncer oral se logra mediante políticas públicas de promoción y prevención en la población, y a través de la promoción de un control odontológico regular.

Los estudios analizados muestran desigualdades substanciales en relación a la prevalencia de patologías de la cavidad oral. Estas desigualdades estaban presentes particularmente en sujetos de bajos ingresos, menor nivel educacional, y en aquellos que vivían en municipalidades rurales. Estos resultados son congruentes con la literatura, y requieren de un abordaje proactivo, que incluya un rango de intervenciones que disminuyan las causas de estas desventajas, y que mejoren la calidad de vida de la población más vulnerable ${ }^{(37)}$.

Lamentablemente, si bien los datos recopilados son diversos, no en todas las ocasiones se realizan las mismas mediciones ni se levanta la misma información, por lo que las comparaciones son difíciles de realizar. Un ejemplo de ello es la falta de uniformidad de la estratificación por grupo de edad, donde la OMS, propone los tramos de edad 35- 44 años y 6574 años para la vigilancia de la patología bucal en la población adulta y adulta mayor ${ }^{(38)}$, así como la falta de uniformidad de la definición de caso en enfermedades periodontales y el uso del CPITN, mencionado anteriormente. Por otro lado, las ENS 2003 y 2016- 2017 definen al personal de enfermería como evaluadores de la salud oral, lo que, en las etapas de capacitación, presentaban una concordancia con el diagnóstico de un odontólogo de un $70 \%$. Al no tener información consistente sobre los puntos anteriores, se dificulta la formulación de políticas públicas adecuadas a las necesidades nacionales.

En nuestro país, si bien se han asegurado algunas prestaciones a través del sistema GES, la prevalencia de enfermedades orales es aún muy alta, y hay sectores que no tienen hasta el momento cobertura como 
son los adultos entre 18 y 59 años, exceptuando a las embarazadas y programas específicos. Tampoco han existido campañas de promoción y prevención de salud oral hacia la población adulta y adulta mayor. Las enfermedades orales raramente son vistas como una prioridad en salud. Es por ello que es necesario que los sistemas odontológicos se enfoquen en la promoción y mantención de la salud oral, para así lograr una mayor equidad en ella. Esto va en congruencia con el llamado a la acción global para una cobertura universal en salud oral, ofreciendo una oportunidad de asegurar el cuidado continuo en niños, adolescentes y en adultos con alto riesgo para mejorar las condiciones orales deterioradas o dañadas ${ }^{(39)}$.

\section{CONCLUSIONES}

Como conclusión podemos decir que los datos sugieren una alta carga de enfermedad periodontal, caries, desdentamiento y lesiones de mucosa oral en adultos y personas mayores chilenas. Esta sinopsis pone de manifiesto la necesidad de diseñar políticas que promuevan condiciones ambientales para facilitar y fortalecer una cultura de prevención y promoción de la salud (prácticas de autocuidado y estilos de vida), que integren la salud bucal dentro de la salud general para conseguir mejores resultados en todos los grupos de edad, disminuyendo las inequidades en salud.

\section{CONFLICTO DE INTERÉS}

Ninguno.

\section{FUENTE DE FINANCIAMIENTO}

Agencia Nacional de Investigación y Desarrollo / Programa Becas / Beca Doctorado Nacional/2018 - 21180023.

\section{RELEVANCIA CLÍNICA}

La recopilación de información epidemiológica de las enfermedades orales a nivel nacional facilita la comunicación y difusión de estos resultados a los clínicos y académicos dedicados a la temática. Se observa una alta prevalencia de enfermedad periodontal, caries, desdentamiento, y lesiones de mucosa oral asociadas al uso de prótesis dental removible en adultos y adultos mayores chilenos, lo que manifiesta la necesidad de diseñar políticas que promuevan condiciones ambientales para facilitar y fortalecer la prevención y promoción de la salud que integren la salud bucal dentro de la salud general para conseguir mejores resultados en todos los grupos de edad.

\section{Bibliografía}

1. Kassebaum NJ, Smith AGC, Bernabé E, Fleming TD, Reynolds AE, Vos T, et al. Global, regional, and national prevalence, incidence, and disability-adjusted life years for oral conditions for 195 countries, 1990-2015: a systematic analysis for the global burden of diseases, injuries, and risk factors. J Dent Res. 2017;96(4):380-7. 2. Bernabe E, Marcenes W, Hernandez CR, Bailey J, Abreu LG, Alipour V, et al. Global, regional, and national levels and trends in burden of oral conditions from 1990 to 2017: a systematic analysis for the global burden of disease 2017 study [Internet]. J Dent Res. 2020;002203452090853. Available from: http://journals. sagepub.com/doi/10.1177/0022034520908533.

3. Broadbent JM, Zeng J, Foster Page LA, Baker SR, Ramrakha S, Thomson WM. Oral health-related beliefs, behaviors, and outcomes through the life course. J Dent Res. 2016;95(7):808-13.

4. Sheiham A. Oral health, general health and quality of life. Bull World Healht Organ. 2005;83(9):644

5. Bray F, Ferlay J, Soerjomataram I, Siegel R, Torre L, Jemal A. Global cancer statistics 2018: GLOBOCAN estimates of incidence and mortality worldwide for 36 cancers in 185 countries. CA Cancer J Clin. 2018;68:394-424.

6. Listl S, Galloway J, Mossey P, Marcenes W. Global economic impact of dental diseases. J Dent Res. 2015;94(10):1355-61.

7. Rawal I, Ghosh S, Hameed SS, Shivashankar R, Ajay VS, Patel SA, et al. Association between poor oral health and diabetes among Indian adult population: Potential for integration with NCDs. BMC Oral Health. 2019;19(1):1-10.

8. Lee J, Oh J, Youk T, Jeong S, Kim Y, Choi S. Association between periodontal disease and non-communicable diseases: A 12-year longitudinal health-examinee cohort study in South Korea. Med. 2017;96(6):e7398.

9. Ministerio de Salud de Chile. Resultados I Encuesta de Salud, Chile 2003 [Internet]. 2016 [consultado 05/12/2019]. Disponible en: http://epi.minsal.cl/wpcontent/uploads/2016/03/InformeFinalENS2003.vent_.pdf.

10. Ministerio de Salud de Chile. Encuesta Nacional de Salud 2016-2017. Segunda entrega de resultados [Internet]. Chile; 2019 [consultado 05/12/2019]. Disponible en: http://epi.minsal.cl/wp-content/uploads/2019/01/2o-Resultados-ENS_DEPTO EPIDEMIOLOGIA.MINSAL.14.01.2019.pdf.

11. Ramírez V, Vásquez-Rozas $P$, Ramírez-Eyraud P. Mortalidad por cáncer oral y faríngeo en Chile, años 2002-2010. Rev Clínica Periodoncia, Implantol y Rehabil Oral. 2015;8(2):133-8.

12. Riera S P, Martínez R B. Morbilidad y mortalidad por cáncer oral y faríngeo en Chile. Rev Med Chil. 2005;133(5):555-63.

13. Gamonal J, Mendoza C, Espinoza I, Muñoz A, Urzúa I, Aranda W, et al Clinical attachment loss in Chilean adult population: First Chilean national dental examination survey. J Periodontol. 2010;81(10):1403-10.

14. Urzúa I, Mendoza C, Arteaga O, Rodríguez G, Cabello R, Faleiros S, et al Dental caries prevalence and tooth loss in Chilean adult population: First national dental examination survey. Int J Dent. 2012;2012.

15. Gamonal JA, Lopez NJ Aranda W. Periodontal conditions and treatment needs, by CPITN, in the 35-44 and 65-74 year-old population in Santiago, Chile. Int Dent J. 1998;48(2):96-103.

16. Espinoza I, Rojas R, Aranda W, Gamonal J. Prevalence of oral mucosal lesions in elderly people in Santiago, Chile. J Oral Pathol Med. 2003;32(10):571-5.

17. Mariño RJ, Cueto A, Badenier R, Acevedo R, Moya R. Oral health status and inequalities among ambulant older adults living in central Chile. Community Dent Health. 2011;28:143-8.

18. Mariño R, Giacaman RA. Factors related to unmet oral health needs in older adults living in Chile. Arch Gerontol Geriatr. 2014;58(3):454-9.

19. Mariño RJ, Fu CS, Giacaman RA. Prevalence of root caries among ambulant older adults living in central Chile. Gerodontology. 2015;32(2):107-14.

20. Giacaman RA, Sandoval D, Bustos IP, Rojas MA. Epidemiología del estado de salud periodontal en la VII. Rev Clin Periodoncia Implant Rehabil Oral. 2016;9(2):184-92.
21. Mariño R, Giacaman RA. Patterns of use of oral health care services and barriers to dental care among ambulatory older Chilean. BMC Oral Health [Internet] 2017;17(1):1-7. Available from: http://dx.doi.org/10.1186/s12903-016-0329-2 22. Ministerio de Salud de Chile. Fe de erratas [Internet]. 2019 [consultado 10/02/2020]. Disponible en: http://epi.minsal.cl/wp-content/uploads/2019/01/Fe-deerratas-2o-Resultados-ENS DEPTO.EPIDEMIOLOGIA.MINSAL.14.01.2019.pdf. 23. Gamonal J, Mendoza C, Espinoza I, Muñoz A, Urzúa I, Aranda W, et al. Clinical attachment aoss in Chilean adult population: First Chilean national dental examination survey. J Periodontol. 2010;81(10):1403-10.

24. Organización Mundial de la Salud. The world health report 1999- making a difference [Internet]. 1999 [cited 25/03/2020]. Available from: https://www.who.int/ whr/1999/en/.

25. Fernández A. II World Assembly on Ageing. Rev Esp Geriatr Gerontol. 2002;37(S2):1-2

26. Jara G, Werlinger F, Cabello R, Carvajal P, Espinoza I, Giacaman R, et al. Sinopsis de la situación de salud oral en Chile - Parte I: garantías explícitas y guías clínicas. Rev clínica periodoncia, Implantol y Rehabil oral. 2018;11(3):187-90.

27. Ministerio de Salud de Chile. Plan Nacional de Salud Bucal 2018-2030 [Internet]. 2017 [consultado 11/02/2020]. Disponible en: https://www.minsal.cl/ wp-content/uploads/2017/12/Plan-Nacional-Salud-Bucal-2018-2030-ConsultaPública-20_12_2017.pdf.

28. Savage A, Eaton K, Moles D, I. N. A systematic review of definitions of periodontitis and methods that have been used to identify this disease. J Clin Periodontol. 2009;36(6):458-67.

29. Martin JA, Grill AC, Matthews AG, Vena D, Thompson VP, Craig RG, et al. Periodontal diagnosis affected by variation in terminology. J Periodontol. 2013;84(5):606-13.

30. Mendoza C, Arteaga O, Gamonal J. Investigación epidemiológica en enfermedades periodontales en América Latina. Rev Chil Period Oseoint [Internet] 2006;3:7-13. Available from: http://scholar.google.com/scholar lookup?title=Investi gación+epidemiológica+en+enfermedad+periodontal+en+América+Latina\&author= Mendoza+C\&author=Arteaga+O\&author=Gamonal+J\&publication_year=2006\&jour nal=Rev+Chil+Period+Oseoint\&volume=3\&pages=7-13\#0.

31. Caton J, Armitage G, Berglundh T, Chapple I, Jepsen S, Kornman K, et al. A new classification scheme for periodontal and peri $\square$ implant diseases and conditions - Introduction and key changes from the 1999 classification. J Clin Periodontol. 2018;45(Suppl 20):S1-8

32. Carvajal P. Enfermedades periodontales como un problema de salud pública: el desafío del nivel primario de atención en salud. Rev Clínica Periodoncia, Implantol y Rehabil Oral [Internet]. 2016;9(2):177-83. Disponible en: http://dx.doi.org/10.1016/j. piro.2016.07.001

33. Morales A, Bravo J, Baeza M, Werlinger F, Gamonal J. Las enfermedades periodontales como enfermedades crónicas no transmisibles: cambios en los paradigmas. Rev Clin Periodoncia Implant Rehabil Oral. 2016;9(2):203-7.

34. Santelices M, Cárcamo M, Brenner C, Montes R. Cáncer oral en Chile. Revisión de la literatura. Rev Med Chil. 2016;144:766-70.

35. Jin L, Lamster I, Greenspan J, Pitts N, Scully C, Warnakulasuriya S. Global burden of oral diseases: emerging concepts, management and interplay with systemic health. Oral Dis. 2016;22(7):609-19.

36. Ministerio de Salud de Chile. Encuesta Nacional de Salud 2016- 2017. Primeros resultados. 2017

37. Espinoza I, Thomson WM, Gamonal J, Arteaga O. Disparities in aspects of oralhealth-related quality of life among Chilean adults. Community Dent Oral Epidemiol. 2013;41(3):242-50

38. Organización Mundial de la Salud. Encuestas de salud bucodental. Métodos básicos. 4a. ed. Ginebra; 1997.

39. Watt RG, Daly B, Allison P, Macpherson LMD, Venturelli R, Listl S, et al. Ending the neglect of global oral health: time for radical action. Lancet. 2019;394(10194):261-72. 